OPEN ACCESS

Edited by:

Amal O. Amer,

The Ohio State University, USA

Reviewed by:

Subramanian Dhandayuthapani, Texas Tech Health Sciences Center,

USA

$L i X U$,

Cornell University, USA

*Correspondence:

Lucile Espert,

CPBS FRE 3689 Centre National de la Recherche Scientifique UM, 1919, route de Mende, 34293 Montpellier,

France

lucile.espert@cpbs.cnrs.fr; Isabelle Vergne,

Institut de Pharmacologie et de Biologie Structurale UMR-5089, BP64182, 205 route de Narbonne, 31077 Toulouse, France isabelle.vergne@ipbs.fr

Received: 30 March 2015 Accepted: 18 May 2015 Published: 02 June 2015

Citation:

Espert L, Beaumelle B and Vergne I (2015) Autophagy in Mycobacterium tuberculosis and HIV infections.

Front. Cell. Infect. Microbiol. 5:49. doi: 10.3389/fcimb.2015.00049

\section{Autophagy in Mycobacterium tuberculosis and HIV infections}

\author{
Lucile Espert $^{1 *}$, Bruno Beaumelle ${ }^{1}$ and Isabelle Vergne ${ }^{2 *}$ \\ ${ }^{1}$ CPBS FRE 3689 Centre National de la Recherche Scientifique, UM, Montpellier, France, ${ }^{2}$ Institut de Pharmacologie et de \\ Biologie Structurale, UMR 5089 Centre National de la Recherche Scientifique - Université de Toulouse, Toulouse, France
}

Human Immunodeficiency Virus (HIV) and Mycobacterium tuberculosis (M.tb) are among the most lethal human pathogens worldwide, each being responsible for around 1.5 million deaths annually. Moreover, synergy between acquired immune deficiency syndrome (AIDS) and tuberculosis (TB) has turned HIV/M.tb co-infection into a major public health threat in developing countries. In the past decade, autophagy, a lysosomal catabolic process, has emerged as a major host immune defense mechanism against infectious agents like M.tb and HIV. Nevertheless, in some instances, autophagy machinery appears to be instrumental for HIV infection. Finally, there is mounting evidence that both pathogens deploy various countermeasures to thwart autophagy. This mini-review proposes an overview of the roles and regulations of autophagy in HIV and M.tb infections with an emphasis on microbial factors. We also discuss the role of autophagy manipulation in the context of HIV/M.tb co-infection. In future, a comprehensive understanding of autophagy interaction with these pathogens will be critical for development of autophagy-based prophylactic and therapeutic interventions for AIDS and TB.

Keywords: Mycobacterium tuberculosis, HIV, AIDS, macrophages, lymphocytes, autophagy, coinfection

\section{Introduction}

Autophagy is an intracellular self-digestion process whereby cytoplasmic constituents are delivered to and degraded by lysosomes (Lamb et al., 2013). Besides being crucial for quality control and energy supply, autophagy plays key roles in immune defenses against invading bacterial and viral pathogens, such as regulation of inflammation, antigen presentation and microorganism capture and degradation (Deretic et al., 2013; Richetta and Faure, 2013; Huang and Brumell, 2014). Autophagy is a tightly regulated process that involves more than 30 dedicated autophagyrelated proteins (Atgs) (Lamb et al., 2013). The process is initiated by two complexes, Ulk1 and Beclin-1, leading to the formation of cup-shaped isolation membrane (Figure 1A). This membrane expands to sequester cytoplasmic target and then closes to form a double-membrane bound organelle termed the autophagosome. Autophagosome biogenesis requires two ubiquitinlike conjugates: Atg12-Atg5 and LC3-phosphatidylethanolamine. Ultimately, autophagosome undergoes fusion with lysosomes leading to the degradation of engulfed material. Cytoplasmic cargo is recognized and then captured by the autophagy machinery via specific LIR domaincontaining autophagic adaptors/receptors that binds directly to LC3 (Deretic et al., 2013). Globally, Ser/Thr kinase mammalian target of rapamycin (mTOR) represses autophagy, but many mTOR-independent pathways can also participate in its regulation (Ravikumar et al., 2010). 


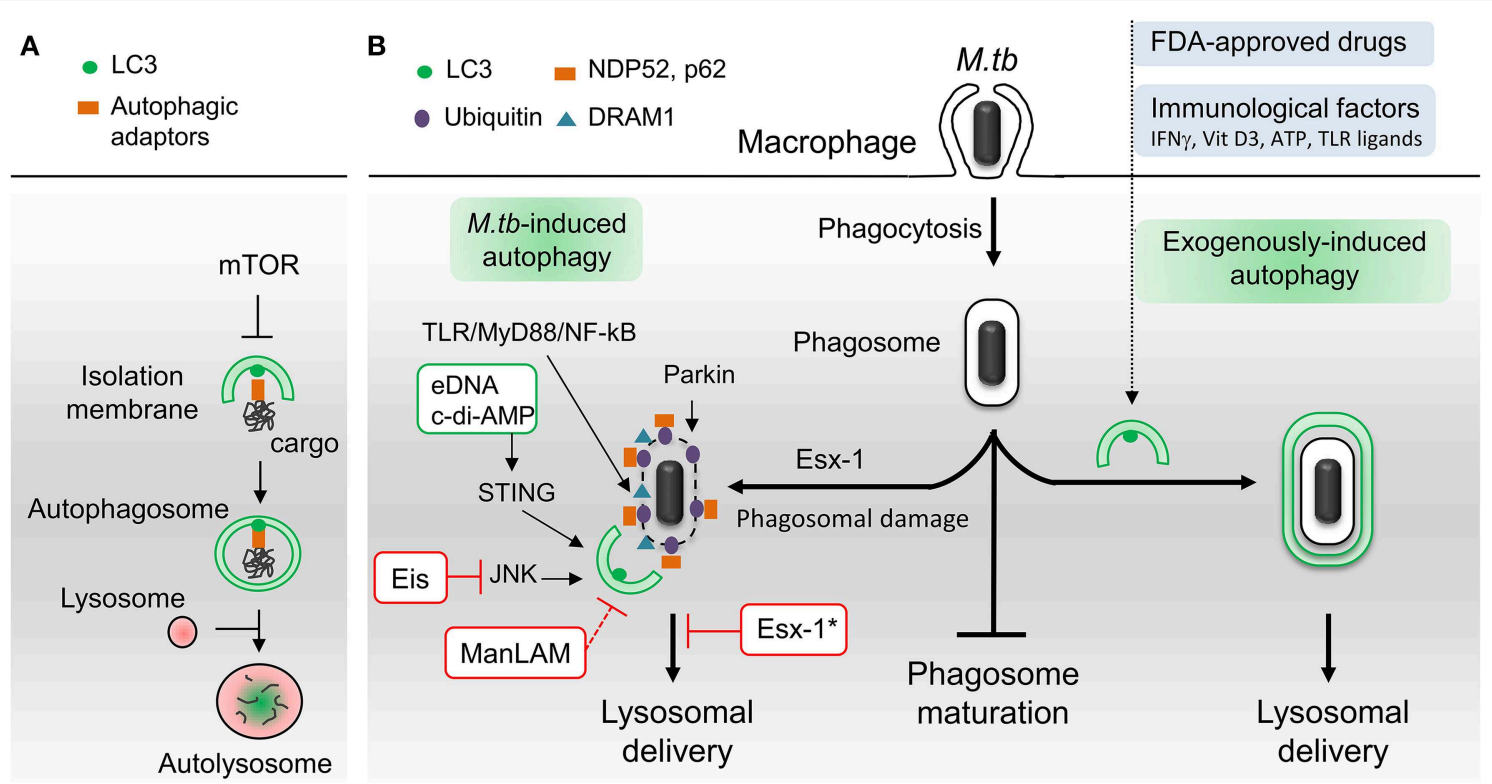

FIGURE 1 | Schematic representation of autophagic pathways and modulators involved in $\boldsymbol{M}$. tuberculosis infection. (A) Principal steps in autophagosome biogenesis and maturation (i.e., Iysosomal delivery). Autophagy begins with the formation of an isolation membrane that grows to enclose cytoplasmic cargo marked with autophagic adaptors. Once sealed, the autophagosome fuses with lysosomes to allow degradation of sequestered cargo. Numerous signaling pathways regulate autophagy including master repressor Ser/Thr kinase mTOR. (B) M.tb-autophagy interaction in macrophage. Following phagocytosis, $M$.tb resides in a vacuole called phagosome and blocks phagosome maturation. Several immunological and pharmaceutical autophagy inducers can restore delivery of $M$.tb to lysosomes. Esx-1-secreting M.tb promotes phagosome damages which trigger ubiquitination, recruitment of autophagic adaptors and mycobacterial capture via STING, Parkin and DRAM1. M.tb extracellular DNA (eDNA) and cyclic-di-adenosine monophosphate (c-di-AMP) activate autophagy whereas Eis and ManLAM inhibit this process. Note that ManLAM was not studied in the context of infection. "Esx-1 blocks autophagosome maturation in human dendritic cells. Vit D3, vitamin D3; TLR, toll-like receptor. Green box, M.tb factors activators of autophagy. Red box, M.tb factors inhibitors of autophagy.

\section{Autophagy in M. tuberculosis Infection and Countermeasures}

The capacity of M.tb to survive and replicate in host macrophages is central to $M . t b$ pathogenesis, with intracellular growth being often associated with virulence (Hmama et al., 2015; Weiss and Schaible, 2015). M.tb has developed several schemes to avoid antimicrobial mechanisms of macrophages and thus survive intracellularly. One of significance is its ability to block phagosome maturation right after phagocytosis thereby avoiding the hostile environment of phagolysosomes and consequently killing (Vergne et al., 2004; Russell, 2011). Importantly, Deretic and collaborators have shown that phagosome maturation and mycobacterial killing can be restored through exogenous induction of autophagy in infected macrophages (Gutierrez et al., 2004).

Since then, several laboratories confirmed this seminal discovery with functionally diverse immunological and pharmacological autophagy inducers, such as IFN $\gamma$, Vitamin D3, ATP, toll-like receptor (TLR) ligands, M.tb-specific T-cells and FDA-approved drugs (Bradfute et al., 2013; Parihar et al., 2014; Schiebler et al., 2014; Stanley et al., 2014) (Figure 1B). Autophagy induction not only results in fusion of lysosomal compartments with mycobacterial phagosome but also triggers the generation of neo-antimicrobial peptides, essential for mycobacterial killing (Alonso et al., 2007; Ponpuak et al., 2010). Moreover, autophagy may play an indirect role in $M . t b$ phagocytosis through regulation of scavenger receptors expression (Bonilla et al., 2013). In addition to these in vitro studies, in vivo experiments have demonstrated that autophagy protein Atg5 in macrophages is required to curtail M.tb infection in mice (Castillo et al., 2012; Watson et al., 2012) and that autophagy inhibitors enhances mycobacterial load in zebrafish embryo model (Van Der Vaart et al., 2014). Notably, several reports revealed that some populations of TB patients display polymorphisms in genes linked to the autophagy pathway, namely, IRGM, IFN $\gamma, I F N \gamma$ receptor, TLR8, Vitamin D3 (VDR), and $A T P$ receptor $(P 2 X 7 R)$, suggesting that some individuals might be more prone to develop TB due to a defective autophagic response (Songane et al., 2012). Current TB treatment involves a cocktail of antimicrobial drugs, among them isoniazid and pyrazinamide. Interestingly, a recent study suggests that these drugs, in addition to their direct bactericidal activity, may also act by promoting autophagy (Kim et al., 2012a). Lastly, in mouse model, increase of autophagy activity during BCG vaccination appears to improve antigen presentation and vaccine efficiency (Jagannath et al., 2009).

Although limited and/or incomplete, autophagy is induced in macrophages as a result of M.tb infection. The molecular mechanisms involved in this activation are just starting to be 
unraveled with two converging signaling pathways described so far (Figure 1B). The first one is a stimulation of interferon genes (STING)-dependent cytosolic DNA sensing pathway that triggers ubiquitination of damaged M.tb-containing phagosome (Watson et al., 2012). Mycobacterial type VII secretion system Esx-1 damages phagosomal membrane and is thus essential to induce this pathway. Both M.tb extracellular DNA (eDNA) and cyclic-di-adenosine monophosphate (c-di-AMP) participate in STING signaling (Watson et al., 2012; Dey et al., 2015). The ensued ubiquitination, mediated in part by ubiquitin ligase Parkin, promotes the recruitment of autophagic adaptors, p62 and NDP52, then LC3 binding and autophagic capture (Watson et al., 2012; Manzanillo et al., 2013). The second is a TLR/MyD88/NF-kB pathway that induces expression of the DNA damage-regulated autophagy modulator DRAM1. DRAM1-induced autophagy requires STING and p62 expression (Van Der Vaart et al., 2014). However, the role of NF-kB in M.tb-induced autophagy is probably complex, since its inhibition was also shown to promote autophagy in infected THP-1 macrophages (Bai et al., 2013). Finally, another layer of regulation may be achieved through expression of microRNA-155 that targets GTPase Rheb, an activator of mTOR (Wang et al., 2013). However, this view has been challenged by another group who showed that M.tb infection enhances mTOR activity (Zullo and Lee, 2012).

In the past 5 years, several reports have revealed that M.tb inhibits autophagy during infection, and that this inhibition could be important for mycobacterial pathogenicity and/or virulence. In contrast to avirulent strain M.tb H37Ra, virulent strain M.tb $\mathrm{H} 37 \mathrm{Rv}$ limits autophagy by upregulating the expression of host anti-autophagic factor Bfl-1/A1 (Kathania et al., 2011). A genome-wide analysis identified additional host genes involved in autophagy inhibition during M.tb H37Rv infection (Kumar et al., 2010). Knockdown of these genes resulted in autophagy-mediated killing of mycobacteria in THP-1 macrophages. Although, some of them have their expression increased during infection, their role in autophagy regulation remains ill-defined. Another host protein important for hampering ubiquitin-dependent autophagic capture of M.tb is Coronin-1a (Seto et al., 2012). To this point, three M.tb products have been shown to suppress autophagy in phagocytes (Figure 1B). A M.tb glycoconjugate, lipoarabinomannan (ManLAM), inhibits autophagy in macrophages by an unknown mechanism (Shui et al., 2011; Vergne et al., 2014). The study was performed using purified molecules and it is thus unclear whether ManLAM is essential for autophagy regulation during infection and if other mycobacterial factors are involved in that process. More recently, the Esx-1secretion system, a M.tb virulence factor, has been implicated in inhibition of autophagic flux in human dendritic cells (Romagnoli et al., 2012). Finally, $M$.tb-secreted Eis is an $\mathrm{N}$-acetyltransferase that blocks autophagy by targeting JNK signaling pathway in murine macrophages (Shin et al., 2010; Kim et al., 2012b).

Overall, these literature data underscore autophagy functionality as a major determinant of M.tb control. Nevertheless, numerous questions remain to be addressed such as the role of autophagy-related proteins and the regulation of autophagy in various M.tb infected-immune and non-immune cells. The molecular mechanisms involved in M.tb manipulation of autophagy needs to be fully characterized as well as the link with $M . t b$ virulence.

\section{Autophagy in HIV Infection and Countermeasures}

HIV-1 infects mainly CD4 T lymphocytes, macrophages and Dendritic Cells (DCs), but its cytopathic effects is mainly characterized by a decline in the CD4 T-cell population (Ameisen and Capron, 1991; Terai et al., 1991). HIV can also access the brain, where it infects and replicates in macrophages, microglial cells, astrocytes and neural precursors, leading to cognitive deficits known as HIV-Associated Neurocognitive Disorders (HAND) (Braathen, 1988; Cavrois et al., 2008; Letendre, 2011).

Several mechanisms are involved in the CD4 T cell demise in HIV-infected patients but, it seems that, although concurrent mechanisms such as pyroptosis have been proposed (Doitsh et al., 2014), most of them die by apoptosis, and that the majority of apoptotic cells are uninfected bystander cells (Ameisen and Capron, 1991; Terai et al., 1991). HIV-1 proteins such as the envelope glycoproteins (Env), the transactivator Tat and the accessory proteins $\mathrm{Vpr}$ and Nef, have been involved in modulating the uninfected CD4 T cell death, but accumulating evidences suggest that Env plays a major role in this process (Varbanov et al., 2006). In this context, infected cells expressing Env at their surface are able to activate autophagy in uninfected CD4 T cells, leading to apoptosis (Espert et al., 2006; Denizot et al., 2008) (Figure 2). While autophagy is induced in uninfected CD4 T cells, it is downregulated in these productively infected cells, suggesting that the virus has evolved strategies to circumvent autophagy antiviral effect (Zhou and Spector, 2008; Espert et al., 2009). Accordingly, a significant increase in autophagy has been detected in the Peripheral Blood Mononuclear Cells of patients that are HIV controllers, i.e., patients that remain asymptomatic for more than 10 years or because they are able to control viremia below the detection limit (Nardacci et al., 2014). At the molecular level, autophagy restricts HIV-1 infection by selectively degrading the viral transactivator Tat but the viral protein Vif is able to inhibit the early steps autophagy allowing the productive infection of CD4 T cells (Borel et al., 2014; Sagnier et al., 2015) (Figure 2). Moreover, rhesus TRIM5 $\alpha$ protein can act as an autophagy receptor since it interacts with the HIV-1 capsid protein and leads to its degradation (Mandell et al., 2014).

Autophagy is not induced in uninfected cells from the monocyte/macrophage lineage after contact with Env-expressing cells (Espert et al., 2009). The secreted form of the HIV-Tat protein could be responsible for the absence of autophagy induction by activating Src-Akt and STAT3 signaling pathways, previously known to inhibit autophagy (Van Grol et al., 2010) (Figure 2). Moreover, the release of Tat by infected cells upregulates IL-10 (Badou et al., 2000) and these two factors can lead to an autophagy blockade in neighboring, uninfected macrophages, independently of the presence of Env 


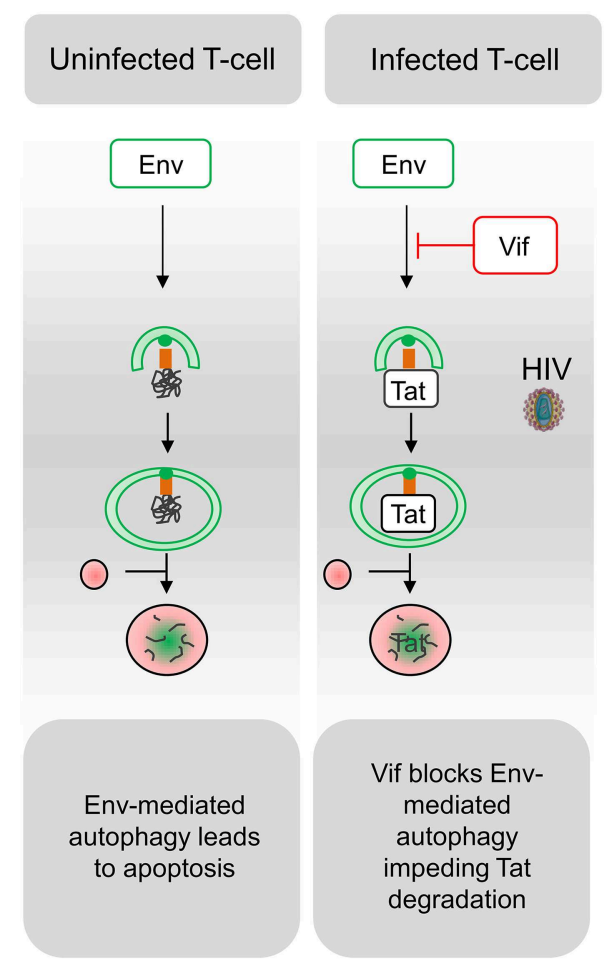

FIGURE 2 | Schematic representation of interactions between HIV and the autophagy pathway. A complex relationship exists between HIV and the autophagy process in the different targeted cell types. Functional autophagy is an anti-HIV process as it promotes degradation of the viral protein Tat, limits virion production and HIV antigen presentation. However, HIV can block the autophagy pathway at the initiation step (Env in dendritic cells; Tat in uninfected

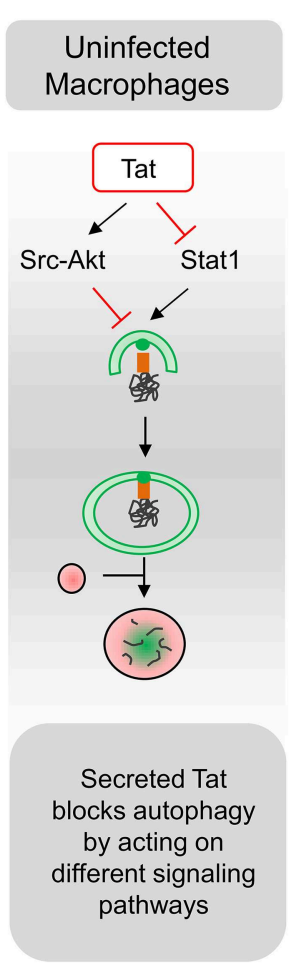

Infected

Dendritic cell

Macrophages

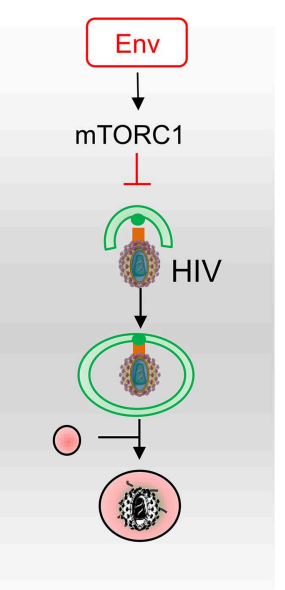

Nef blocks HIV-1induced autophagy at the autophagosome maturation step to enhance viral yields
Env inhibits autophagy via mTOR to blocks presentation of HIV antigens to $T$ cells
(Van Grol et al., 2010). Tat also suppresses IFN- $\gamma$-induced autophagy in macrophages by suppressing Stat 1 phosphorylation (Li et al., 2011). Conversely, autophagosomes are accumulated in infected macrophages where Nef, by interaction with Beclin 1, blocks autophagy before its degradative step (Espert et al., 2009; Kyei et al., 2009). The HIV-1 precursor Gag was found in complexes with LC3 and was also present in LC3-IIenriched membranes, suggesting that autophagy could favor Gag processing and viral particles production in these cells (Kyei et al., 2009). Notably, the degradative step of autophagy has an anti-HIV-1 activity that must be controlled by the virus to prevent its degradation (Figure 2). Nef also interacts with the cellular immunity-associated GTPase family M (IRGM) promoting autophagosomes accumulation and improving HIV replication. The anti-HIV activity of autophagy is also evidenced by reports showing that TLR8 ligands activate a vitamin D3mediated autophagic response that inhibits HIV-1 multiplication in macrophages (Campbell and Spector, 2011, 2012b).

The viral protein ASP (AntiSens Protein), which is produced from a transcript initiating from the promoter-harboring 3'long terminal repeat region of the viral genome, has been shown to partially co-localize with LC3 (Clerc et al., 2011; Torresilla et al., 2013). ASP expression could induce autophagy macrophages; Vif in infected CD4 T cells) or at the maturation step (Nef in infected macrophages). Notably, the blockade of autophagy maturation by Nef, in infected macrophages, can be beneficial for HIV. In uninfected CD4 T cells, Env-induced autophagy is a prerequisite for the induction of apoptosis. Viral determinants that are inducers of autophagy are indicated in green, and viral determinants that inhibit this process are indicated in red. in the promonocytic U937 cells and, consequently, increase viral replication. ASP could also be a target for autophagic degradation, allowing the regulation of its expression as it was already demonstrated for the Human T-Lymphtropic Virus (HTLV) protein Tax (Tang et al., 2013; Torresilla et al., 2013).

In DCs, the viral replication intermediates could be transported into the lysosome in an autophagy-dependent manner and regulate IFN- $\alpha$ secretion upon TLR7-mediated ssRNA recognition (Lee et al., 2007; Zhou et al., 2012). Moreover, autophagy inhibition has been shown to impair TLR-mediated innate immune response while also strongly affecting antigen processing and MHC-II-mediated antigen presentation to CD4 T cells (Blanchet et al., 2010) (Figure 2).

HIV can invade the central nervous system and autophagy has been proposed to participate in HAND but the exact mechanisms of the HIV-mediated alteration of the autophagic pathway is poorly understood. Autophagic vacuoles accumulation has been observed in post-mortem brain from patients with HIVassociated encephalitis (Zhou et al., 2011). Notably, autophagy is a key pathway regulating aging and HIV patients developing HAND have symptoms usually associated with an aged brain and increased susceptibility to neuropathologies (Fields et al., 2013). 
Importantly, several studies demonstrated that the ATGs can exert some effects independently of the autophagic process (Bestebroer et al., 2013). Indeed, while it becomes obvious that autophagy has an anti-HIV effect, a genome-wide RNAi screen has demonstrated that some ATG proteins are required for HIV1 replication (Brass et al., 2008). Accordingly, it has been shown that HIV-1 replication can be delayed in stable ATG knockdown cell lines (Eekels et al., 2012). These studies highlighted a role of autophagy separated from the effect of ATG proteins alone during HIV infection.

In conclusion, the autophagy/HIV relationship is complex and depends mainly on the targeted cell types and their infectious status (Figure 2). In the future, it would be interesting to decipher the Env-induced signaling pathways leading to autophagydependent apoptosis and to study autophagy regulation in different cohort of patients in parallel to in vitro experiments. This is of particular importance considering that bystander CD4 T lymphocytes constitute the majority of dying cells during HIV infection, leading to AIDS. Because autophagy is an anti-HIV process blocked by the virus for its replication, the development of drugs inducing this process could be beneficial for the patients.

\section{Autophagy in TB/HIV Co-infections}

Synergy between HIV-1 and M.tb is a very severe problem. Each pathogen favors infection by the other. Indeed, M.tb is responsible for $26 \%$ of deaths in HIV-infected patients, and $39 \%$ of new M.tb cases concern HIV-1 positive patients (Harrington, 2010; Chang et al., 2013). This deadly association is especially critical in sub-saharan Africa and a warning has been launched "When will we act" (Harries et al., 2010).

It is largely unknown how infection by HIV-1 facilitates infection by M.tb (Anandaiah et al., 2011; Diedrich and Flynn, 2011). Nevertheless, the development of HIV-1 infection strongly affects the immune system and thus prevents the development of an efficient cellular response against M.tb (Chang et al., 2013). The main target of HIV-1 is the CD4+ T-cell (Perelson et al., 1996) and only $<10 \%$ of macrophages are infected in patients (Kedzierska et al., 2003; Sandler and Douek, 2012). This is due to a combination of several factors such as low expression of HIV-1 receptor (CD4) and coreceptor (CXCR5) on macrophages (Alexaki et al., 2008) and the expression in this cell type of SAMHD1 that is able to inhibit HIV-1 multiplication (Hrecka et al., 2011). Nevertheless, the macrophage infection rate by HIV1 is substantially increased upon opportunistic infection and doubly infected macrophages have been observed within the lymph nodes of a patient co-infected with HIV-1 and M.avium (Orenstein et al., 1997) and in alveolar macrophages from a patient co-infected with HIV-1 and M.tb (Mwandumba et al., 2004). Upon coinfection of the same macrophage, inhibition of the autophagy pathway by the first invader will likely benefit the second.

More generally, it seems clear that manipulation of the immune response by HIV-1 and M.tb favors the multiplication of the other pathogen. For instance, the induction of IL-10 secretion by HIV-1 (Badou et al., 2000; Rayne et al., 2004) was found to inhibit apoptosis of macrophages upon M.tb infection (Patel et al., 2009). More globally, this immunosuppressive Th2 cytokine will hamper the cellular immune response to M.tb (Jasenosky et al., 2015). In the other direction, M.tb infection was found to increase IL-8 levels (Friedland et al., 1992) and this cytokine, by inducing the homing of T-cells to lymph nodes, will favor HIV-1 multiplication (Ott et al., 1998).

A key effector allowing the immune system to fight both HIV-1 and M.tb is vitamin D. It is produced in the skin through exposure to ultraviolet $\mathrm{B}$. It is considered that half of the human population have vitamin $\mathrm{D}$ insufficiency and this deficiency is much more widespread and pronounced in HIV1 infected patients (Borella et al., 2014). The active metabolite of vitamin $\mathrm{D}$ is $1 \alpha, 25$-dihydroxy-cholecalciferol $(1,25 \mathrm{D} 3)$ which is produced by the kidney and proinflammatory cells, in particular during infection. Upon binding to its receptor, $1,25 \mathrm{D} 3$ will enhance the expression of $\beta$-defensin $2 / 4$ and $4 \mathrm{~A}$ genes as well as cathelicidin (Liu et al., 2006; Borella et al., 2014). These peptides are mediators of antibacterial and antiviral responses, and cathelicidin induces the transcription of ATG5, Beclin1 and NOD2 that are key components of the autophagy pathway (Yuk et al., 2009; Borella et al., 2014).

$1,25 \mathrm{D} 3$ is thus an important physiological inducer of autophagy, a process that is involved in cell defense against both HIV-1 and M.tb. (Deretic, 2012). Indeed 1,25D3 was found to inhibit both HIV-1 and M.tb multiplication in an autophagy-dependent manner (Campbell and Spector, 2012c; Anandaiah et al., 2013). Inhibition of HIV-1 or $M . t b$ growth by physiological levels of $1,25 \mathrm{D} 3$ is dependent on Beclin-1 and ATG5, and this is also the case when macrophages are infected with both pathogens (Campbell and Spector, 2012c). Vitamin D-induced autophagy was also found to rely on cathelicidin to eliminate HIV-1 and $M . t b$ either alone or in coinfection (Campbell and Spector, 2012c).

The capacity of vitamin D to induce autophagy and inhibit the growth of HIV-1 and M.tb has been reviewed in detail elsewhere (Jo, 2010; Campbell and Spector, 2012a). Although supplementation of vitamin D led to contrasting results it seems clear that a normal level of this vitamin helps the immune system to fight several type of bacterial and viral infections (Borella et al., 2014).

\section{Conclusions and Perspectives}

Here, we have gathered compelling published data that reveal the major functions and regulation mechanisms of autophagy in HIV and M.tb infections. However, despite considerable progress, our understanding of autophagy in these deadly infections remains largely incomplete. An in-depth comprehension of the intricate interplay between HIV, M.tb and autophagy, both in vitro and in vivo, will undoubtedly unveil novel strategies employed by pathogens to subvert this pathway. Furthermore, this research avenue could also lead to improved knowledge of the autophagy pathway itself and to innovative tools for 
autophagy manipulation, as it was recently achieved with NefBeclin-1 interaction (Kyei et al., 2009; Shoji-Kawata et al., 2013). Although several challenges have to be overcome, in particular the multiple and complexes roles of autophagy in infection and cellular homeostasis, autophagy manipulation holds great potentials for future development of new or improved vaccines and therapies for infectious diseases (Espert et al., 2006; Kyei et al., 2009; Rubinsztein et al., 2012; Mostowy, 2013; Levine et al., 2015; Wallis and Hafner, 2015).

\section{References}

Alexaki, A., Liu, Y., and Wigdahl, B. (2008). Cellular reservoirs of HIV1 and their role in viral persistence. Curr. HIV Res. 6, 388-400. doi: $10.2174 / 157016208785861195$

Alonso, S., Pethe, K., Russell, D. G., and Purdy, G. E. (2007). Lysosomal killing of Mycobacterium mediated by ubiquitin-derived peptides is enhanced by autophagy. Proc. Natl. Acad. Sci. U.S.A. 104, 6031-6036. doi: 10.1073/pnas.0700036104

Ameisen, J. C., and Capron, A. (1991). Cell dysfunction and depletion in AIDS: the programmed cell death hypothesis. Immunol. Today 12, 102-105. doi: 10.1016/0167-5699(91)90092-8

Anandaiah, A., Dheda, K., Keane, J., Koziel, H., Moore, D. A., and Patel, N. R. (2011). Novel developments in the epidemic of human immunodeficiency virus and tuberculosis coinfection. Am. J. Respir. Crit. Care Med. 183, 987-997. doi: 10.1164/rccm.201008-1246CI

Anandaiah, A., Sinha, S., Bole, M., Sharma, S. K., Kumar, N., Luthra, K., et al. (2013). Vitamin D rescues impaired Mycobacterium tuberculosis-mediated tumor necrosis factor release in macrophages of HIV-seropositive individuals through an enhanced Toll-like receptor signaling pathway in vitro. Infect. Immun. 81, 2-10. doi: 10.1128/IAI.00666-12

Badou, A., Bennasser, Y., Moreau, M., Leclerc, C., Benkirane, M., and Bahraoui, E. (2000). Tat protein of human immunodeficiency virus type 1 induces interleukin-10 in human peripheral blood monocytes: implication of protein kinase C-dependent pathway. J. Virol. 74, 10551-10562. doi: 10.1128/JVI.74.22.10551-10562.2000

Bai, X., Feldman, N. E., Chmura, K., Ovrutsky, A. R., Su, W. L., Griffin, L., et al. (2013). Inhibition of nuclear factor-kappa B activation decreases survival of Mycobacterium tuberculosis in human macrophages. PLoS ONE 8:e61925. doi: 10.1371/journal.pone.0061925

Bestebroer, J., V'Kovski, P., Mauthe, M., and Reggiori, F. (2013). Hidden behind autophagy: the unconventional roles of ATG proteins. Traffic 14, 1029-1041. doi: 10.1111/tra.12091

Blanchet, F. P., Moris, A., Nikolic, D. S., Lehmann, M., Cardinaud, S., Stalder, R., et al. (2010). Human immunodeficiency virus-1 inhibition of immunoamphisomes in dendritic cells impairs early innate and adaptive immune responses. Immunity 32, 654-669. doi: 10.1016/j.immuni.2010.04.011

Bonilla, D. L., Bhattacharya, A., Sha, Y., Xu, Y., Xiang, Q., Kan, A., et al. (2013). Autophagy regulates phagocytosis by modulating the expression of scavenger receptors. Immunity 39, 537-547. doi: 10.1016/j.immuni.2013.08.026

Borel, S., Robert-Hebmann, V., Alfaisal, J., Jain, A., Faure, M., Espert, L., et al. (2014). HIV-1 viral infectivity factor interacts with light chain 3 and inhibits autophagy. AIDS. 29, 275-286.

Borella, E., Nesher, G., Israeli, E., and Shoenfeld, Y. (2014). Vitamin D: a new anti-infective agent? Ann. N.Y. Acad. Sci. 1317, 76-83. doi: 10.1111/nyas.12321

Braathen, L. R. (1988). Langerhans cells and HIV infection. Biomed. Pharmacother. 42, 305-308.

Bradfute, S. B., Castillo, E. F., Arko-Mensah, J., Chauhan, S., Jiang, S., Mandell, M., et al. (2013). Autophagy as an immune effector against tuberculosis. Curr. Opin. Microbiol. 16, 355-365. doi: 10.1016/j.mib.2013.05.003

Brass, A. L., Dykxhoorn, D. M., Benita, Y., Yan, N., Engelman, A., Xavier, R. J., et al. (2008). Identification of host proteins required for HIV infection through a functional genomic screen. Science 319, 921-926. doi: 10.1126/science.1152725

Campbell, G. R., and Spector, S. A. (2011). Hormonally active vitamin D3 (1alpha,25-dihydroxycholecalciferol) triggers autophagy in human

\section{Acknowledgments}

The work in the authors' laboratories was supported by EU FP7 Marie Curie Career Integration Grant 293416 (I.Vergne), University of Toulouse III (IV), the organization "Vaincre la mucoviscidose" (IV), EU Horizon 2020 TBVAC2020 (IV), Centre National de la Recherche Scientifique (CNRS), Montpellier University (UM) (BB and LE), Sidaction (BB and LE) and the Agence Nationale de Recherche sur le SIDA (ANRS) (BB and LE).

macrophages that inhibits HIV-1 infection. J. Biol. Chem. 286, 18890-18902. doi: 10.1074/jbc.M110.206110

Campbell, G. R., and Spector, S. A. (2012a). Autophagy induction by vitamin D inhibits both Mycobacterium tuberculosis and human immunodeficiency virus type 1. Autophagy 8, 1523-1525. doi: 10.4161/auto.21154

Campbell, G. R., and Spector, S. A. (2012b). Toll-like receptor 8 ligands activate a vitamin $\mathrm{D}$ mediated autophagic response that inhibits human immunodeficiency virus type 1. PLoS Pathog. 8:e1003017. doi: 10.1371/journal.ppat.1003017

Campbell, G. R., and Spector, S. A. (2012c). Vitamin D inhibits human immunodeficiency virus type 1 and Mycobacterium tuberculosis infection in macrophages through the induction of autophagy. PLoS Pathog. 8:e1002689. doi: 10.1371/journal.ppat.1002689

Castillo, E. F., Dekonenko, A., Arko-Mensah, J., Mandell, M. A., Dupont, N., Jiang, S., et al. (2012). Autophagy protects against active tuberculosis by suppressing bacterial burden and inflammation. Proc. Natl. Acad. Sci. U.S.A. 109, E3168-E3176. doi: 10.1073/pnas.1210500109

Cavrois, M., Neidleman, J., and Greene, W. C. (2008). The achilles heel of the trojan horse model of HIV-1 trans-infection. PLoS Pathog. 4:e1000051. doi: 10.1371/journal.ppat.1000051

Chang, C. C., Crane, M., Zhou, J., Mina, M., Post, J. J., Cameron, B. A., et al. (2013). HIV and co-infections. Immunol. Rev. 254, 114-142. doi: 10.1111/imr. 12063

Clerc, I., Laverdure, S., Torresilla, C., Landry, S., Borel, S., Vargas, A., et al. (2011). Polarized expression of the membrane ASP protein derived from HIV1 antisense transcription in $\mathrm{T}$ cells. Retrovirology 8:74. doi: 10.1186/17424690-8-74

Denizot, M., Varbanov, M., Espert, L., Robert-Hebmann, V., Sagnier, S., Garcia, E., et al. (2008). HIV-1 gp41 fusogenic function triggers autophagy in uninfected cells. Autophagy 4, 998-1008. doi: 10.4161/auto.6880

Deretic, V. (2012). Autophagy as an innate immunity paradigm: expanding the scope and repertoire of pattern recognition receptors. Curr. Opin. Immunol. 24, 21-31. doi: 10.1016/j.coi.2011.10.006

Deretic, V., Saitoh, T., and Akira, S. (2013). Autophagy in infection, inflammation and immunity. Nat. Rev. Immunol. 13, 722-737. doi: 10.1038/nri3532

Dey, B., Dey, R. J., Cheung, L. S., Pokkali, S., Guo, H., Lee, J. H., et al. (2015). A bacterial cyclic dinucleotide activates the cytosolic surveillance pathway and mediates innate resistance to tuberculosis. Nat. Med. 21, 401-406. doi: $10.1038 / \mathrm{nm} .3813$

Diedrich, C. R., and Flynn, J. L. (2011). HIV-1/mycobacterium tuberculosis coinfection immunology: how does HIV-1 exacerbate tuberculosis? Infect. Immun. 79, 1407-1417. doi: 10.1128/iai.01126-10

Doitsh, G., Galloway, N. L., Geng, X., Yang, Z., Monroe, K. M., Zepeda, O., et al. (2014). Cell death by pyroptosis drives CD4 T-cell depletion in HIV-1 infection. Nature 505, 509-514. doi: 10.1038/nature12940

Eekels, J. J., Sagnier, S., Geerts, D., Jeeninga, R. E., Biard-Piechaczyk, M., and Berkhout, B. (2012). Inhibition of HIV-1 replication with stable RNAimediated knockdown of autophagy factors. Virol. J. 9:69. doi: 10.1186/1743422X-9-69

Espert, L., Denizot, M., Grimaldi, M., Robert-Hebmann, V., Gay, B., Varbanov, M., et al. (2006). Autophagy is involved in T cell death after binding of HIV-1 envelope proteins to CXCR4. J. Clin. Invest. 116, 2161-2172. doi: 10.1172/JCI26185

Espert, L., Varbanov, M., Robert-Hebmann, V., Sagnier, S., Robbins, I., Sanchez, F., et al. (2009). Differential role of autophagy in CD4 $\mathrm{T}$ cells and 
macrophages during X4 and R5 HIV-1 infection. PLoS ONE 4:e5787. doi: 10.1371/journal.pone.0005787

Fields, J., Dumaop, W., Rockenstein, E., Mante, M., Spencer, B., Grant, I., et al. (2013). Age-dependent molecular alterations in the autophagy pathway in HIVE patients and in a gp120 tg mouse model: reversal with beclin-1 gene transfer. J. Neurovirol. 19, 89-101. doi: 10.1007/s13365-012-0145-7

Friedland, J. S., Remick, D. G., Shattock, R., and Griffin, G. E. (1992). Secretion of interleukin-8 following phagocytosis of Mycobacterium tuberculosis by human monocyte cell lines. Eur. J. Immunol. 22, 1373-1378. doi: 10.1002/eji.1830220607

Gutierrez, M. G., Master, S. S., Singh, S. B., Taylor, G. A., Colombo, M. I., and Deretic, V. (2004). Autophagy is a defense mechanism inhibiting BCG and Mycobacterium tuberculosis survival in infected macrophages. Cell 119, 753-766. doi: 10.1016/j.cell.2004.11.038

Harries, A. D., Zachariah, R., Corbett, E. L., Lawn, S. D., Santos-Filho, E. T., Chimzizi, R., et al. (2010). The HIV-associated tuberculosis epidemic-when will we act? Lancet 375, 1906-1919. doi: 10.1016/S0140-6736(10)60409-6

Harrington, M. (2010). From HIV to tuberculosis and back again: a tale of activism in 2 pandemics. Clin. Infect. Dis. 50(Suppl. 3), S260-S266. doi: 10.1086/651500

Hmama, Z., Pena-Diaz, S., Joseph, S., and Av-Gay, Y. (2015). Immunoevasion and immunosuppression of the macrophage by Mycobacterium tuberculosis. Immunol. Rev. 264, 220-232. doi: 10.1111/imr.12268

Hrecka, K., Hao, C., Gierszewska, M., Swanson, S. K., Kesik-Brodacka, M., Srivastava, S., et al. (2011). Vpx relieves inhibition of HIV-1 infection of macrophages mediated by the SAMHD1 protein. Nature 474, 658-661. doi: $10.1038 /$ nature 10195

Huang, J., and Brumell, J. H. (2014). Bacteria-autophagy interplay: a battle for survival. Nat. Rev. Microbiol. 12, 101-114. doi: 10.1038/nrmicro3160

Jagannath, C., Lindsey, D. R., Dhandayuthapani, S., Xu, Y., Hunter, R. L. Jr., and Eissa, N. T. (2009). Autophagy enhances the efficacy of BCG vaccine by increasing peptide presentation in mouse dendritic cells. Nat. Med. 15, 267-276. doi: $10.1038 / \mathrm{nm} .1928$

Jasenosky, L. D., Scriba, T. J., Hanekom, W. A., and Goldfeld, A. E. (2015). T cells and adaptive immunity to Mycobacterium tuberculosis in humans. Immunol. Rev. 264, 74-87. doi: 10.1111/imr.12274

Jo, E.-K. (2010). Innate immunity to mycobacteria: vitamin D and autophagy. Cell. Microbiol. 12, 1026-1035. doi: 10.1111/j.1462-5822.2010.01491.x

Kathania, M., Raje, C. I., Raje, M., Dutta, R. K., and Majumdar, S. (2011). Bfl-1/A1 acts as a negative regulator of autophagy in mycobacteria infected macrophages. Int. J. Biochem. Cell Biol. 43, 573-585. doi: 10.1016/j.biocel.2010.12.014

Kedzierska, K., Azzam, R., Ellery, P., Mak, J., Jaworowski, A., and Crowe, S. M. (2003). Defective phagocytosis by human monocyte/macrophages following HIV-1 infection: underlying mechanisms and modulation by adjunctive cytokine therapy. J. Clin. Virol. 26, 247-263. doi: 10.1016/S13866532(02)00123-3

Kim, J. J., Lee, H. M., Shin, D. M., Kim, W., Yuk, J. M., Jin, H. S., et al. (2012a). Host cell autophagy activated by antibiotics is required for their effective antimycobacterial drug action. Cell Host Microbe 11, 457-468. doi: 10.1016/j.chom.2012.03.008

Kim, K. H., An, D. R., Song, J., Yoon, J. Y., Kim, H. S., Yoon, H. J., et al. (2012b). Mycobacterium tuberculosis Eis protein initiates suppression of host immune responses by acetylation of DUSP16/MKP-7. Proc. Natl. Acad. Sci. U.S.A. 109, 7729-7734. doi: 10.1073/pnas.1120251109

Kumar, D., Nath, L., Kamal, M. A., Varshney, A., Jain, A., Singh, S., et al. (2010). Genome-wide analysis of the host intracellular network that regulates survival of Mycobacterium tuberculosis. Cell 140, 731-743. doi: $10.1016 /$ j.cell.2010.02.012

Kyei, G. B., Dinkins, C., Davis, A. S., Roberts, E., Singh, S. B., Dong, C., et al. (2009). Autophagy pathway intersects with HIV-1 biosynthesis and regulates viral yields in macrophages. J. Cell Biol. 186, 255-268. doi: 10.1083/jcb.200903070

Lamb, C. A., Yoshimori, T., and Tooze, S. A. (2013). The autophagosome: origins unknown, biogenesis complex. Nat. Rev. Mol. Cell Biol. 14, 759-774. doi: $10.1038 / \mathrm{nrm} 3696$

Lee, H. K., Lund, J. M., Ramanathan, B., Mizushima, N., and Iwasaki, A. (2007). Autophagy-dependent viral recognition by plasmacytoid dendritic cells. Science 315, 1398-1401. doi: 10.1126/science. 1136880

Letendre, S. (2011). Central nervous system complications in HIV disease: HIVassociated neurocognitive disorder. Top. Antivir. Med. 19, 137-142.
Levine, B., Packer, M., and Codogno, P. (2015). Development of autophagy inducers in clinical medicine. J. Clin. Invest. 125, 14-24. doi: 10.1172/ JCI73938

Li, J. C., Au, K. Y., Fang, J. W., Yim, H. C., Chow, K. H., Ho, P. L., et al. (2011). HIV-1 trans-activator protein dysregulates IFN-gamma signaling and contributes to the suppression of autophagy induction. AIDS 25, 15-25. doi: 10.1097/QAD.0b013e328340fd61

Liu, P. T., Stenger, S., Li, H., Wenzel, L., Tan, B. H., Krutzik, S. R., et al. (2006). Toll-like receptor triggering of a vitamin D-mediated human antimicrobial response. Science 311, 1770-1773. doi: 10.1126/science.1123933

Mandell, M. A., Jain, A., Arko-Mensah, J., Chauhan, S., Kimura, T., Dinkins, C., et al. (2014). TRIM proteins regulate autophagy and can target autophagic substrates by direct recognition. Dev. Cell 30, 394-409. doi: 10.1016/j.devcel.2014.06.013

Manzanillo, P. S., Ayres, J. S., Watson, R. O., Collins, A. C., Souza, G., Rae, C. S., et al. (2013). The ubiquitin ligase parkin mediates resistance to intracellular pathogens. Nature 501, 512-516. doi: 10.1038/nature12566

Mostowy, S. (2013). Autophagy and bacterial clearance: a not so clear picture. Cell. Microbiol. 15, 395-402. doi: 10.1111/cmi.12063

Mwandumba, H. C., Russell, D. G., Nyirenda, M. H., Anderson, J., White, S. A., Molyneux, M. E., et al. (2004). Mycobacterium tuberculosis resides in nonacidified vacuoles in endocytically competent alveolar macrophages from patients with tuberculosis and HIV infection. J. Immunol. 172, 4592-4598. doi: 10.4049/jimmunol.172.7.4592

Nardacci, R., Amendola, A., Ciccosanti, F., Corazzari, M., Esposito, V., Vlassi, C., et al. (2014). Autophagy plays an important role in the containment of HIV-1 in nonprogressor-infected patients. Autophagy 10, 1167-1178. doi: 10.4161/auto. 28678

Orenstein, J. M., Fox, C., and Wahl, S. M. (1997). Macrophages as a source of HIV during opportunistic infections. Science 276, 1857-1861. doi: $10.1126 /$ science.276.5320.1857

Ott, M., Lovett, J. L., Mueller, L., and Verdin, E. (1998). Superinduction of IL-8 in T cells by HIV-1 Tat protein is mediated through NF-kappaB factors. J. Immunol. 160, 2872-2880.

Parihar, S. P., Guler, R., Khutlang, R., Lang, D. M., Hurdayal, R., Mhlanga, M. M., et al. (2014). Statin therapy reduces the mycobacterium tuberculosis burden in human macrophages and in mice by enhancing autophagy and phagosome maturation. J. Infect. Dis. 209, 754-763. doi: 10.1093/infdis/jit550

Patel, N. R., Swan, K., Li, X., Tachado, S. D., and Koziel, H. (2009). Impaired M. tuberculosis-mediated apoptosis in alveolar macrophages from HIV+ persons: potential role of IL-10 and BCL-3. J. Leukoc. Biol. 86, 53-60. doi: 10.1189/jlb.0908574

Perelson, A. S., Neumann, A. U., Markowitz, M., Leonard, J. M., and Ho, D. D. (1996). HIV-1 dynamics in vivo: virion clearance rate, infected cell life span, and viral generation time. Science 271, 1582-1586. doi: $10.1126 /$ science.271.5255.1582

Ponpuak, M., Davis, A. S., Roberts, E. A., Delgado, M. A., Dinkins, C., Zhao, Z., et al. (2010). Delivery of cytosolic components by autophagic adaptor protein p62 endows autophagosomes with unique antimicrobial properties. Immunity 32, 329-341. doi: 10.1016/j.immuni.2010.02.009

Ravikumar, B., Sarkar, S., Davies, J. E., Futter, M., Garcia-Arencibia, M., Green-Thompson, Z. W., et al. (2010). Regulation of mammalian autophagy in physiology and pathophysiology. Physiol. Rev. 90, 1383-1435. doi: 10.1152/physrev.00030.2009

Rayne, F., Vendeville, A., Bonhoure, A., and Beaumelle, B. (2004). The ability of chloroquine to prevent tat-induced cytokine secretion by monocytes is implicated in its in vivo anti-human immunodeficiency virus type 1 activity. J. Virol. 78, 12054-12057. doi: 10.1128/JVI.78.21.1205412057.2004

Richetta, C., and Faure, M. (2013). Autophagy in antiviral innate immunity. Cell. Microbiol. 15, 368-376. doi: 10.1111/cmi.12043

Romagnoli, A., Etna, M. P., Giacomini, E., Pardini, M., Remoli, M. E., Corazzari, M., et al. (2012). ESX-1 dependent impairment of autophagic flux by Mycobacterium tuberculosis in human dendritic cells. Autophagy 8, 1357-1370. doi: 10.4161 /auto.20881

Rubinsztein, D. C., Codogno, P., and Levine, B. (2012). Autophagy modulation as a potential therapeutic target for diverse diseases. Nat. Rev. Drug Discov. 11, 709-730. doi: $10.1038 /$ nrd 3802 
Russell, D. G. (2011). Mycobacterium tuberculosis and the intimate discourse of a chronic infection. Immunol. Rev. 240, 252-268. doi: 10.1111/j.1600065X.2010.00984.x

Sagnier, S., Daussy, C. F., Borel, S., Robert-Hebmann, V., Faure, M., Blanchet, F. P., et al. (2015). Autophagy restricts HIV-1 infection by selectively degrading Tat in CD4+ T lymphocytes. J. Virol. 89, 615-625. doi: 10.1128/JVI.02174-14

Sandler, N. G., and Douek, D. C. (2012). Microbial translocation in HIV infection: causes, consequences and treatment opportunities. Nat. Rev. Microbiol. 10, 655-666. doi: 10.1038/nrmicro2848

Schiebler, M., Brown, K., Hegyi, K., Newton, S. M., Renna, M., Hepburn, L., et al. (2014). Functional drug screening reveals anticonvulsants as enhancers of mTOR-independent autophagic killing of Mycobacterium tuberculosis through inositol depletion. EMBO Mol. Med. 7, 127-139. doi: $10.15252 / \mathrm{emmm} .201404137$

Seto, S., Tsujimura, K., and Koide, Y. (2012). Coronin-1a inhibits autophagosome formation around Mycobacterium tuberculosis-containing phagosomes and assists mycobacterial survival in macrophages. Cell. Microbiol. 14, 710-727. doi: 10.1111/j.1462-5822.2012.01754.X

Shin, D. M., Jeon, B. Y., Lee, H. M., Jin, H. S., Yuk, J. M., Song, C. H., et al. (2010). Mycobacterium tuberculosis eis regulates autophagy, inflammation, and cell death through redox-dependent signaling. PLoS Pathog. 6:e1001230. doi: 10.1371/journal.ppat.1001230

Shoji-Kawata, S., Sumpter, R., Leveno, M., Campbell, G. R., Zou, Z., Kinch, L., et al. (2013). Identification of a candidate therapeutic autophagy-inducing peptide. Nature 494, 201-206. doi: 10.1038/nature11866

Shui, W., Petzold, C. J., Redding, A., Liu, J., Pitcher, A., Sheu, L., et al. (2011). Organelle membrane proteomics reveals differential influence of mycobacterial lipoglycans on macrophage phagosome maturation and autophagosome accumulation. J. Proteome Res. 10, 339-348. doi: 10.1021/pr100688h

Songane, M., Kleinnijenhuis, J., Netea, M. G., and van Crevel, R. (2012). The role of autophagy in host defence against Mycobacterium tuberculosis infection. Tuberculosis (Edinb). 92, 388-396. doi: 10.1016/j.tube.2012.05.004

Stanley, S. A., Barczak, A. K., Silvis, M. R., Luo, S. S., Sogi, K., Vokes, M., et al. (2014). Identification of host-targeted small molecules that restrict intracellular Mycobacterium tuberculosis growth. PLoS Pathog. 10:e1003946. doi: 10.1371/journal.ppat.1003946

Tang, S. W., Chen, C. Y., Klase, Z., Zane, L., and Jeang, K. T. (2013). The cellular autophagy pathway modulates human T-cell leukemia virus type 1 replication. J. Virol. 87, 1699-1707. doi: 10.1128/JVI.02147-12

Terai, C., Kornbluth, R. S., Pauza, C. D., Richman, D. D., and Carson, D. A. (1991). Apoptosis as a mechanism of cell death in cultured T lymphoblasts acutely infected with HIV-1. J. Clin. Invest. 87, 1710-1715. doi: 10.1172/JCI115188

Torresilla, C., Larocque, E., Landry, S., Halin, M., Coulombe, Y., Masson, J. Y., et al. (2013). Detection of the HIV-1 minus-strand-encoded antisense protein and its association with autophagy. J. Virol. 87, 5089-5105. doi: 10.1128/JVI. 00225-13

Van Der Vaart, M., Korbee, C. J., Lamers, G. E., Tengeler, A. C., Hosseini, R., Haks, M. C., et al. (2014). The DNA damage-regulated autophagy modulator DRAM1 links mycobacterial recognition via TLP-MYD88 to authophagic defense. Cell Host Microbe 15, 753-767. doi: 10.1016/j.chom.2014.05.005
Van Grol, J., Subauste, C., Andrade, R. M., Fujinaga, K., Nelson, J., and Subauste, C. S. (2010). HIV-1 inhibits autophagy in bystander macrophage/monocytic cells through Src-Akt and STAT3. PLoS ONE 5:e11733. doi: 10.1371/journal.pone.0011733

Varbanov, M., Espert, L., and Biard-Piechaczyk, M. (2006). Mechanisms of CD4 T-cell depletion triggered by HIV-1 viral proteins. AIDS Rev. 8, 221-236.

Vergne, I., Chua, J., Singh, S. B., and Deretic, V. (2004). Cell biology of mycobacterium tuberculosis phagosome. Annu. Rev. Cell Dev. Biol. 20, 367-394. doi: 10.1146/annurev.cellbio.20.010403.114015

Vergne, I., Gilleron, M., and Nigou, J. (2014). Manipulation of the endocytic pathway and phagocyte functions by Mycobacterium tuberculosis lipoarabinomannan. Front. Cell. Infect. Microbiol. 4:187. doi: 10.3389/fcimb.2014.00187

Wallis, R. S., and Hafner, R. (2015). Advancing host-directed therapy for tuberculosis. Nat. Rev. Immunol. 15, 255-263. doi: 10.1038/nri3813

Wang, J., Yang, K., Zhou, L., Minhaowu, Wu. Y., Zhu, M., et al. (2013). MicroRNA155 promotes autophagy to eliminate intracellular mycobacteria by targeting Rheb. PLoS Pathog. 9:e1003697. doi: 10.1371/journal.ppat.1003697

Watson, R. O., Manzanillo, P. S., and Cox, J. S. (2012). Extracellular M. tuberculosis DNA targets bacteria for autophagy by activating the host DNA-sensing pathway. Cell 150, 803-815. doi: 10.1016/j.cell.2012.06.040

Weiss, G., and Schaible, U. E. (2015). Macrophage defense mechanisms against intracellular bacteria. Immunol. Rev. 264, 182-203. doi: 10.1111/imr.12266

Yuk, J.-M., Shin, D.-M., Lee, H.-M., Yang, C.-S., Jin, H. S., Kim, K.-K., et al. (2009). Vitamin D3 induces autophagy in human monocytes/macrophages via cathelicidin. Cell Host Microbe 6, 231-243. doi: 10.1016/j.chom.2009.08.004

Zhou, D., Kang, K. H., and Spector, S. A. (2012). Production of interferon alpha by human immunodeficiency virus type 1 in human plasmacytoid dendritic cells is dependent on induction of autophagy. J. Infect. Dis. 205, 1258-1267. doi: $10.1093 /$ infdis/jis 187

Zhou, D., Masliah, E., and Spector, S. A. (2011). Autophagy is increased in postmortem brains of persons with HIV-1-associated encephalitis. J. Infect. Dis. 203, 1647-1657. doi: 10.1093/infdis/jir163

Zhou, D., and Spector, S. A. (2008). Human immunodeficiency virus type-1 infection inhibits autophagy. AIDS 22, 695-699. doi: 10.1097/QAD.0b013e3282f4a836

Zullo, A. J., and Lee, S. (2012). Mycobacterial induction of autophagy varies by species and occurs independently of mammalian target of rapamycin inhibition. J. Biol. Chem. 287, 12668-12678. doi: 10.1074/jbc.M111.320135

Conflict of Interest Statement: The authors declare that the research was conducted in the absence of any commercial or financial relationships that could be construed as a potential conflict of interest.

Copyright (c) 2015 Espert, Beaumelle and Vergne. This is an open-access article distributed under the terms of the Creative Commons Attribution License (CC BY). The use, distribution or reproduction in other forums is permitted, provided the original author(s) or licensor are credited and that the original publication in this journal is cited, in accordance with accepted academic practice. No use, distribution or reproduction is permitted which does not comply with these terms. 